\title{
AiMT
}

Advances in Military Technology

Vol. 15, No. 2, 2020, pp. 343-353

ISSN 1802-2308, eISSN 2533-4123

DOI 10.3849/aimt.01403

\section{A Motion Model of a Complex-Shaped Remotely Operated Underwater Vehicle}

\author{
D. Thai Nguyen ${ }^{1}$, V. Horák ${ }^{2 *}$, H. Thu Tran ${ }^{3}$, L. The Nguyen ${ }^{1}$ \\ and C. Quang Hoang ${ }^{1}$ \\ ${ }^{1}$ Le Quy Don Technical University, Hanoi, Vietnam \\ ${ }^{2}$ University of Defence in Brno, Czech Republic \\ ${ }^{3}$ Institute of Mechanics, Hanoi, Vietnam
}

\begin{abstract}
The manuscript was received on 6 April 2020 and was accepted after revision for publication as research paper on 21 September 2020.
\end{abstract}

\begin{abstract}
:
The knowledge of velocities of a remotely operated underwater vehicle (ROV) is crucial for the study of the ROV motion. The ROV motion equations are complemented by hydrodynamic parameters and forces acting upon the ROV. The matrices of hydrodynamic damping coefficients and external forces acting upon the ROV are considered in this study as well. The computational results obtained by the Runge-Kutta method are compared with the experiment. It appears that the presented model can be useful for the design and investigation of remotely operated underwater vehicles.
\end{abstract}

\section{Keywords:}

mathematical model, motion equation, remotely operated underwater vehicle, RungeKutta method, water flow

\section{Introduction}

Remotely operated underwater vehicles are widely used for underwater survey missions such as the location, positioning and identification of various submerged structures and objects. ROVs have been used for inspection works, for example, pipeline surveys, jacket inspections and marine hull inspection of vessels. They are also used to assist with the exploration of ocean resources and with the protection of marine environment. The military use of ROVs is primarily for naval mine-hunting and mine-breaking.

There are numerous research studies in the field of underwater vehicles in the world. Many investigations of underwater vehicles motion assume the body geometry

\footnotetext{
* Corresponding author: University of Defence in Brno, Kounicova 156/65, CZ-662 10 Brno, Czech Republic. Phone: +420 973442 616, E-mail: vladimir.horak@unob.cz. ORCID 00000003-1654-7441.
} 
having three planes of symmetry that substantially simplifies the mathematical model of motion. For example, Gomes et al. [1] studied the ROV motion of three planes of symmetry at the University of Porto. Likewise, Marzbanrad et al. [2] applied the three planes of symmetry for design and control of ROV, built in the Shiraz University Robotic Lab. In addition, Eidsvik and Schjølberg [3] from the Norwegian University of Science and Technology used three planes of symmetry for the evaluation of hydrodynamic parameters that characterize their ROV behaviour. In these studies, the matrices of ROV hydrodynamic damping coefficients are diagonal.

In this study, the research is focused on the complex-shaped ROV with two planes of symmetry and one asymmetric plane. The solution results for the ROV motion are then compared with the experiment. The configuration of the examined ROV, consisting of the main body, two lights, two thrusters, and two balance flotation blocks is shown in Fig. 1.

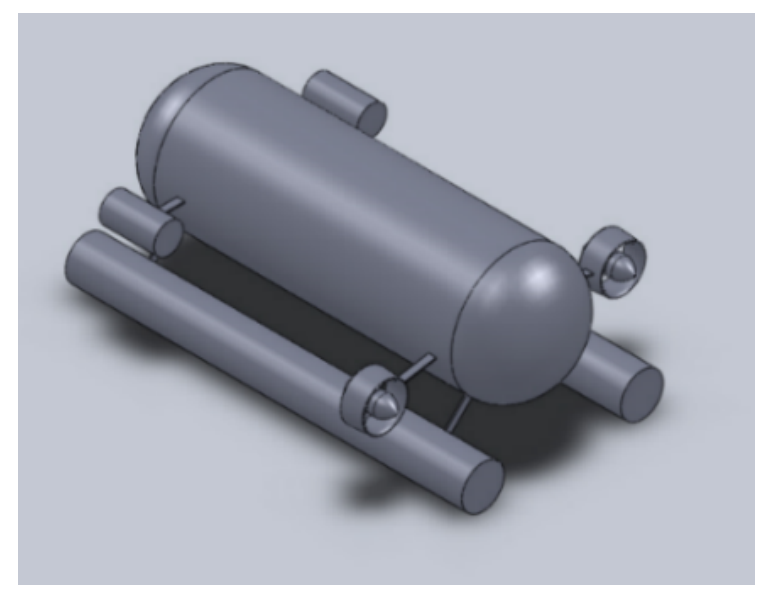

Fig. 1 Concept of $3 D$ ROV model

\section{Mathematical Model of the ROV Motion}

The developed mathematical model provides solutions for velocities, hydrodynamic parameters and forces acting upon the ROV.

\subsection{Coordinate Systems}

There are two coordinate systems used to describe the ROV motion: the earth-fixed coordinate system $\left(O_{0}, X_{0}, Y_{0}, Z_{0}\right)$ and the ROV body-fixed coordinate system $(O, X, Y$, $Z$ ). Both of the coordinate systems are shown in Fig. 2.

Here:

- $\boldsymbol{\eta}_{1}=[x, y, z]^{\mathrm{T}}$ is the position vector and $\boldsymbol{\eta}_{2}=[\phi, \theta, \psi]^{\mathrm{T}}$ is the orientation vector of the ROV in the earth-fixed coordinate system $\left(O_{0}, X_{0}, Y_{0}, Z_{0}\right)$. Operator T refers to the matrix transformation,

- $\boldsymbol{\tau}_{1}=[X, Y, Z]^{\mathrm{T}}$ and $\boldsymbol{\tau}_{2}=[K, M, N]^{\mathrm{T}}$ are the total forces and momenta acting on the ROV in the body-fixed coordinate system $(O, X, Y, Z)$,

- $\boldsymbol{V}=\left[\boldsymbol{v}_{1}, \boldsymbol{v}_{2}\right]^{\mathrm{T}}=[u, v, w, p, q, r]^{\mathrm{T}}$, where $\boldsymbol{v}_{1}=[u, v, w]^{\mathrm{T}}$ and $\boldsymbol{v}_{2}=[p, q, r]^{\mathrm{T}}$ are the translational and rotational velocities of the ROV in the body-fixed coordinate system $(O, X, Y, Z)$. 


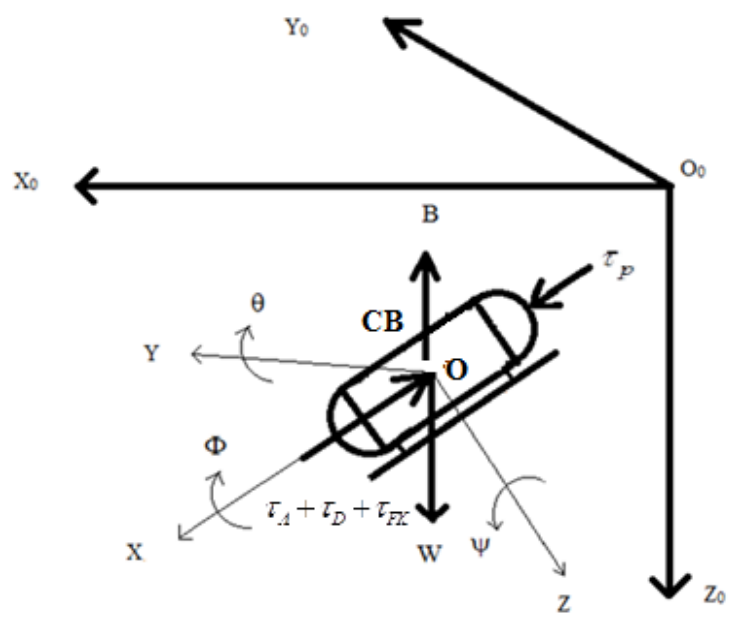

Fig. 2 Earth-fixed $\left(O_{0}, X_{0}, Y_{0}, Z_{0}\right)$ and body-fixed $(O, X, Y, Z)$ coordinate systems

\subsection{Model Assumptions}

To reduce the model complexity, the following simplifying assumptions are made:

- ROV has two planes of symmetry: port-starboard plane, fore-aft plane,

- ROV is a rigid body of constant mass,

- ROV is deeply submerged in a homogeneous unbounded fluid,

- the effects of surface waves, underwater walls and bottom are ignored,

- the linear and angular couple of terms in damping is neglected,

- the damping terms of the second and higher orders are neglected.

The fore-aft plane symmetry is admitted due to the geometric similarity of lights and thrusters.

\subsection{Model of the ROV Motion}

The first coordinate transform relates to translational velocities of the ROV between the two coordinate systems [4]:

$$
\left[\begin{array}{c}
\dot{x} \\
\dot{y} \\
\dot{z}
\end{array}\right]=\boldsymbol{J}_{1}\left(\boldsymbol{\eta}_{2}\right)\left[\begin{array}{c}
u \\
v \\
w
\end{array}\right]
$$

with

$$
\boldsymbol{J}_{1}\left(\boldsymbol{\eta}_{2}\right)=\left[\begin{array}{ccc}
\cos \psi \cos \theta & -\sin \psi \cos \phi+\cos \psi \sin \theta \sin \phi & \sin \psi \sin \phi+\cos \psi \sin \theta \cos \phi \\
\sin \psi \cos \theta & \cos \psi \cos \phi+\sin \psi \sin \theta \sin \phi & -\cos \psi \sin \phi+\sin \psi \sin \theta \cos \phi \\
-\sin \theta & \cos \theta \sin \phi & \cos \theta \cos \phi
\end{array}\right] \text { (2) }
$$

The second coordinate transform relates to rotational velocities of the ROV between the two coordinate systems [5]:

$$
\left[\begin{array}{c}
\dot{\phi} \\
\dot{\theta} \\
\dot{\psi}
\end{array}\right]=\boldsymbol{J}_{2}\left(\boldsymbol{\eta}_{2}\right)\left[\begin{array}{c}
p \\
q \\
r
\end{array}\right]
$$


with

$$
J_{2}\left(\boldsymbol{\eta}_{2}\right)=\left[\begin{array}{ccc}
1 & \sin \phi \tan \theta & \cos \phi \tan \theta \\
0 & \cos \phi & -\sin \phi \\
0 & \frac{\sin \phi}{\cos \theta} & \frac{\cos \phi}{\sin \theta}
\end{array}\right]
$$

The relative velocity between the ROV and the surrounding water flow is defined as follows:

$$
\boldsymbol{V}_{r}=\left[\boldsymbol{V}_{\boldsymbol{r}_{1}}, \boldsymbol{V}_{\boldsymbol{r}_{2}}\right]^{\mathrm{T}}=\boldsymbol{V}-\boldsymbol{V}_{\text {water }}
$$

where $\boldsymbol{V}_{r_{1}}=\left[u_{r}, v_{r}, w_{r}\right]^{\mathrm{T}}, \boldsymbol{V}_{r_{2}}=\left[p_{r}, q_{r}, r_{r}\right]^{\mathrm{T}}$ and $\boldsymbol{V}_{\text {water }}=\left[u_{l}, v_{l}, w_{l}, 0,0,0\right]^{\mathrm{T}}$.

The set of equations describing the ROV motion can be written as follows [3]:

$$
\boldsymbol{M}_{\mathrm{RB}} \dot{\boldsymbol{V}}+\boldsymbol{C}_{\mathrm{RB}}(\boldsymbol{V}) \boldsymbol{V}=\boldsymbol{\tau}
$$

where

- $\boldsymbol{M}_{\mathrm{RB}}$ is the generalized mass matrix of the ROV,

- $\boldsymbol{C}_{\mathrm{RB}}$ is the Coriolis force and central force matrix,

- $\tau$ are the total forces and momenta acting upon the ROV.

$$
\boldsymbol{\tau}=\left[\boldsymbol{\tau}_{1}, \boldsymbol{\tau}_{2}\right]^{\mathrm{T}}=\boldsymbol{\tau}_{\mathrm{HS}}+\boldsymbol{\tau}_{\mathrm{A}}+\boldsymbol{\tau}_{\mathrm{D}}+\boldsymbol{\tau}_{\mathrm{FK}}+\boldsymbol{\tau}_{\mathrm{P}}
$$

where

- $\boldsymbol{\tau}_{\mathrm{HS}}$ is the restoring force and moment vector,

- $\tau_{\mathrm{D}}$ is the damping force and moment vector,

- $\boldsymbol{\tau}_{\mathrm{A}}$ is the added mass force and moment vector,

- $\tau_{\mathrm{FK}}$ is the Froude-Krylov force vector,

- $\boldsymbol{\tau}_{\mathrm{P}}$ is the thruster force from two propellers vector.

The restoring force and moment vector $\boldsymbol{\tau}_{\mathrm{HS}}$ is given by the following formula:

$$
\boldsymbol{\tau}_{\mathrm{HS}}=\left[\begin{array}{c}
-(W-B) \sin \theta \\
(W-B) \cos \theta \sin \phi \\
(W-B) \cos \theta \cos \phi \\
\left(y_{G} W-y_{B} B\right) \cos \theta \cos \phi-\left(z_{G} W-z_{B} B\right) \cos \theta \sin \phi \\
-\left(z_{G} W-z_{B} B\right) \sin \theta-\left(x_{G} W-x_{B} B\right) \cos \theta \cos \phi \\
\left(x_{G} W-x_{B} B\right) \cos \theta \sin \phi-\left(y_{G} W-y_{B} B\right) \sin \theta
\end{array}\right]
$$

here

- $W=m g$ is the gravity of the ROV at the mass centre,

- $B=\rho g V_{\mathrm{ROV}}$ is the buoyancy force of the ROV at the buoyancy centre,

- $g$ is the acceleration due to gravity,

- $V_{\mathrm{ROV}}$ is the ROV volume,

- $m$ is the ROV mass in the air,

- $\rho$ is the water density,

- $x_{b}, y_{b}, z_{b}$ and $x_{G}, y_{G}, z_{G}$ are the positions of the centre of buoyancy and the centre of gravity with respect to the origin coordinate, respectively.

The damping force and moment $\tau_{\mathrm{D}}$ is given by the following formula [5]: 


$$
\boldsymbol{\tau}_{\mathrm{D}}=\boldsymbol{D}\left(\boldsymbol{V}_{r}\right) \boldsymbol{V}_{r}
$$

where $\boldsymbol{D}\left(\boldsymbol{V}_{r}\right)$ is the hydrodynamic damping matrix that is written as [3]:

$$
\boldsymbol{D}\left(\boldsymbol{V}_{r}\right)=\left[\begin{array}{cccccc}
X_{u|u|} \cdot|u r| & 0 & 0 & 0 & 0 & 0 \\
0 & Y_{v|v|} \cdot|v r| & 0 & 0 & 0 & 0 \\
0 & 0 & Z_{w|w|} \cdot|w r| & 0 & 0 & 0 \\
0 & K_{v|v|} \cdot|v r| & 0 & K_{p|p|} \cdot\left|p_{r}\right| & 0 & 0 \\
M_{u|u|} \cdot|u r| & 0 & 0 & 0 & M_{q|q|} \cdot\left|q_{r}\right| & 0 \\
0 & 0 & 0 & 0 & 0 & N_{r|r|} \cdot|r r|
\end{array}\right]
$$

here $X_{u|u|}, Y_{v|v|}, Z_{w|w|}, K_{p|p|}, M_{q|q|}, N_{r|r|}, K_{v|v|}, M_{u|u|}$ are the quadratic damping coefficients, which may be calculated by using the following formula $[6,7]$ :

$$
X_{u|u|}=-\frac{1}{2} \rho c_{\mathrm{d}} A_{f x}, Y_{v|v|}=-\frac{1}{2} \rho c_{\mathrm{dc}} A_{f y}, Z_{w|w|}=-\frac{1}{2} \rho c_{\mathrm{dc}} A_{f z}
$$

where

- $\rho$ is the density of the surrounding water,

- $A_{f x}, A_{f y}, A_{f z}$ is the ROV frontal area along $O X, O Y, O Z$,

- $c_{\mathrm{d}}$ is the axial drag coefficient of the ROV,

- $c_{\mathrm{dc}}$ is the crossflow drag coefficient of the ROV,

- $K_{p|p|}, M_{q|q|}, N_{r|r|}, K_{v|v|}, M_{u|u|}$ are the damping moment coefficients of the ROV, that can be calculated by using the strip theory [6].

The added mass force and moment $\tau_{\mathrm{A}}$ can be calculated by the following formula [3]:

$$
\boldsymbol{\tau}_{\mathrm{A}}=-\left[\boldsymbol{M}_{\mathrm{A}} \dot{\boldsymbol{V}}_{r}+\boldsymbol{C}_{\mathrm{A}}\left(\boldsymbol{V}_{r}\right) \boldsymbol{V}_{r}\right]
$$

where $\boldsymbol{M}_{\mathrm{A}}$ and $\boldsymbol{C}_{\mathrm{A}}\left(\boldsymbol{V}_{r}\right)$ are the added mass and Coriolis-like matrix induced by $M_{\mathrm{A}}$, respectively.

Under the assumptions made, the ROV has two planes of symmetry (portstarboard plane, fore-aft plane) and one asymmetric plane (bottom-top plane). The added mass matrix is calculated as follows [5]:

$$
\boldsymbol{M}_{\mathrm{A}}=-\left[\begin{array}{cccccc}
X_{\dot{u}} & 0 & 0 & 0 & X_{\dot{q}} & 0 \\
0 & Y_{\dot{v}} & 0 & Y_{\dot{p}} & 0 & 0 \\
0 & 0 & Z_{\dot{w}} & 0 & 0 & 0 \\
0 & K_{\dot{v}} & 0 & K_{\dot{p}} & 0 & 0 \\
M_{\dot{u}} & 0 & 0 & 0 & M_{\dot{q}} & 0 \\
0 & 0 & 0 & 0 & 0 & N_{\dot{r}}
\end{array}\right]=\left[\begin{array}{cccccc}
m_{11} & 0 & 0 & 0 & m_{15} & 0 \\
0 & m_{22} & 0 & m_{24} & 0 & 0 \\
0 & 0 & m_{33} & 0 & 0 & 0 \\
0 & m_{42} & 0 & m_{44} & 0 & 0 \\
m_{51} & 0 & 0 & 0 & m_{55} & 0 \\
0 & 0 & 0 & 0 & 0 & m_{66}
\end{array}\right]
$$

where $m_{i j}$ is the added mass coefficient of ROV in the $i$ direction caused by the ROV acceleration in the $j$ direction; $i, j=1,2,3,4,5,6$.

Based on the work by Fossen [8], the off-diagonal elements of a positive inertia matrix are much smaller than the diagonal counterparts. Therefore, the off-diagonal elements in the added mass matrix (13) can be neglected.

The Coriolis-like matrix $\boldsymbol{C}_{\mathrm{A}}\left(\boldsymbol{V}_{r}\right)$ is written as follows [5]: 


$$
\boldsymbol{C}_{\mathrm{A}}\left(\boldsymbol{V}_{r}\right)=\left[\begin{array}{cccccc}
0 & 0 & 0 & 0 & Z_{\dot{\mathrm{w}}} w_{r} & -Y_{\dot{v}} v_{r} \\
0 & 0 & 0 & -Z_{\dot{\mathrm{w}}} w_{r} & 0 & X_{\dot{u}} u_{r} \\
0 & 0 & 0 & Y_{\dot{v}} v_{r} & -X_{\dot{u}} u_{r} & 0 \\
0 & Z_{\dot{\mathrm{w}}} w_{r} & -Y_{\dot{v}} v_{r} & 0 & N_{\dot{r}} r_{r} & -M_{\dot{q}} q_{r} \\
-Z_{\dot{\mathrm{w}}} w_{r} & 0 & X_{\dot{u}} u_{r} & -N_{\dot{r}} r_{r} & 0 & K_{\dot{p}} p_{r} \\
Y_{\dot{v}} v_{r} & -X_{\dot{u}} u_{r} & 0 & M_{\dot{q}} q_{r} & -K_{\dot{p}} p_{r} & 0
\end{array}\right]
$$

The Froude-Krylov force $\boldsymbol{\tau}_{\mathrm{FK}}$ appears due to the inertia matrix of the displaced fluid. This force is defined $[3,8]$ by

$$
\boldsymbol{\tau}_{\mathrm{FK}}=\boldsymbol{M}_{\mathrm{FK}} \dot{\boldsymbol{V}}_{\mathrm{water}}
$$

where $\boldsymbol{M}_{\mathrm{FK}}$ is the Froude-Krylov mass of the ROV. According to the study by Khalid Isa [4], the $\boldsymbol{M}_{\mathrm{FK}}$ can be considered equal to the mass of the ROV that simplifies the solution

$$
\boldsymbol{M}_{F K}=\boldsymbol{M}_{\mathrm{RB}}
$$
formula:

The thruster force from the two propellers can be calculated by the following

$$
\boldsymbol{\tau}_{\mathrm{P}}=\left[X_{\mathrm{P}}, Y_{\mathrm{P}}, Z_{\mathrm{P}}, K_{\mathrm{P}}, M_{\mathrm{P}}, N_{\mathrm{P}}\right]^{\mathrm{T}}
$$

here:

- $X_{\mathrm{P}}, Y_{\mathrm{P}}, Z_{\mathrm{P}}$ are the forces from the two propellers along $O X, O Y, O Z$,

- $\quad K_{\mathrm{P}}, M_{\mathrm{P}}, N_{\mathrm{P}}$ are the momenta from the two propellers around $O X, O Y, O Z$.

By substituting Eqs (7), (9), (12) into Eq. (6), the set of equations describing the ROV motion can be rewritten in the form

$$
\left[\boldsymbol{M}_{\mathrm{RB}}+\boldsymbol{M}_{\mathrm{A}}\right] \dot{\boldsymbol{V}}_{r}=-\boldsymbol{C}_{\mathrm{RB}}(\boldsymbol{V}) \boldsymbol{V}-\boldsymbol{C}_{\mathrm{A}}\left(\boldsymbol{V}_{r}\right) \boldsymbol{V}_{r}+\boldsymbol{D}\left(\boldsymbol{V}_{r}\right) \boldsymbol{V}_{r}+\boldsymbol{\tau}_{\mathrm{HS}}+\boldsymbol{\tau}_{\mathrm{P}}
$$

After some algebra, Eq. (18) can be rewritten as follows:

$$
\dot{\boldsymbol{V}}=\dot{\boldsymbol{V}}_{\text {water }}+\boldsymbol{M}^{-1} \boldsymbol{A}\left(\boldsymbol{V}, \boldsymbol{V}_{r}\right)
$$

where:

$$
\boldsymbol{A}\left(\boldsymbol{V}, \boldsymbol{V}_{r}\right)=\left[-\boldsymbol{C}_{\mathrm{RB}}(\boldsymbol{V}) \boldsymbol{V}-\boldsymbol{C}_{\mathrm{A}}\left(\boldsymbol{V}_{r}\right) \boldsymbol{V}_{r}+\boldsymbol{D}\left(\boldsymbol{V}_{r}\right) \boldsymbol{V}_{r}+\boldsymbol{\tau}_{\mathrm{HS}}+\boldsymbol{\tau}_{\mathrm{P}}\right]
$$

and

$$
\boldsymbol{M}=\boldsymbol{M}_{\mathrm{RB}}+\boldsymbol{M}_{\mathrm{A}}
$$

\section{Validation of the ROV Motion Model}

The mathematical model described above has been solved by the Runge-Kutta method using the Fortran programing language. The input parameters to achieve the solution are given in Tab. 1. The solving process ran for 20 seconds with the time step of 0.002 seconds.

Some solution results for the ROV motion are shown in Figs 3-6. The evolutions of the ROV velocity components are shown in Fig. 3. The component of velocity vector $V_{x}$ along the $O_{0} X_{0}$ axis is shown in Fig. 3a. One can see that the velocity $V_{x}$ is invariable after about 5 seconds of moving, as its value reaches $1.65 \mathrm{~m} / \mathrm{s}$. The evolution of the velocity component $V_{z}$ along the $O_{0} Z_{0}$ axis is shown in Fig. 3b. This value 
oscillates around zero. After about 10 seconds, the ROV movement reaches a quasisteady state. However, the velocity component $V_{y}$ along $O_{0} Y_{0}$ equals zero at all times, because the propulsive forces from both of the propellers are equal. These results agree well with real observations.

Tab. 1 Model input parameters

\begin{tabular}{|l|c|c|c|}
\hline \multicolumn{1}{|c|}{ Input parameter } & Symbol & Unit & Value \\
\hline Mass of the ROV & $m$ & $\mathrm{~kg}$ & 42.5 \\
\hline Length of the ROV & $L$ & $\mathrm{~m}$ & 0.8 \\
\hline Diameter of the ROV main body & $D$ & $\mathrm{~m}$ & 0.25 \\
\hline Moment of inertia & $I_{x x}$ & $\mathrm{~kg} \mathrm{~m}^{2}$ & 0.81 \\
\hline Moment of inertia & $I_{y y}$ & $\mathrm{~kg} \mathrm{~m}^{2}$ & 2.73 \\
\hline Moment of inertia & $I_{z z}$ & $\mathrm{~kg} \mathrm{~m}^{2}$ & 2.62 \\
\hline Moments of inertia & $I_{x y}, I_{y z}, I_{z x}$ & $\mathrm{~kg} \mathrm{~m}^{2}$ & 0 \\
\hline Length of the light & $l_{\text {light }}$ & $\mathrm{m}$ & 0.11 \\
\hline Diameter of the ligth & $d_{\text {light }}$ & $\mathrm{m}$ & 0.06 \\
\hline Length of the thruster & $l_{\mathrm{DC}}$ & $\mathrm{m}$ & 0.102 \\
\hline Diameter of the ligth & $d_{\mathrm{DC}}$ & $\mathrm{m}$ & 0.097 \\
\hline Length of the balance block & $l_{\mathrm{bb}}$ & $\mathrm{m}$ & 0.8 \\
\hline Diameter of the balance block & $d_{\mathrm{bb}}$ & $\mathrm{m}$ & 0.086 \\
\hline Density of fluid & $\rho$ & $\mathrm{kg} / \mathrm{m}^{3}$ & 1000 \\
\hline Initial velocity of water flow & $V_{\mathrm{water}}$ & $\mathrm{m} / \mathrm{s}$ & 0 \\
\hline Initial velocity of ROV & $V_{0}$ & $\mathrm{~m} / \mathrm{s}$ & 0 \\
\hline Initial position of ROV & $x_{0}, y_{0}$ & $\mathrm{~m}$ & 0 \\
\hline Initial depth of ROV & $z_{0}$ & $\mathrm{~m}$ & 1 \\
\hline Initial orientation of ROV & $\phi_{0}, \theta_{0}, \psi_{0}$ & $\mathrm{rad}$ & 0 \\
\hline Force from each thruster & $\tau_{\mathrm{P}}$ & $\mathrm{N}$ & 20 \\
\hline
\end{tabular}

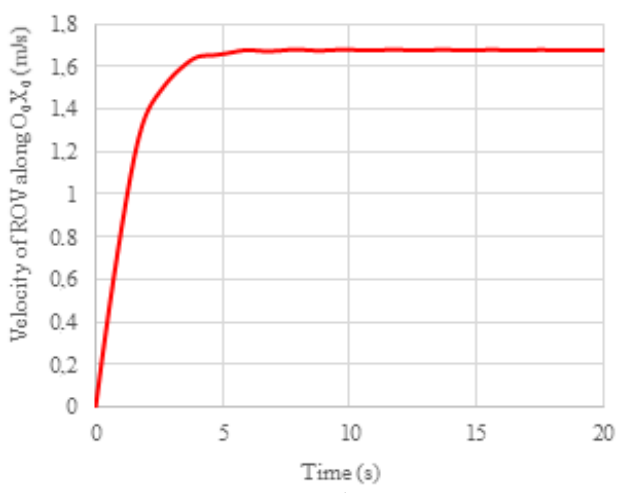

a)

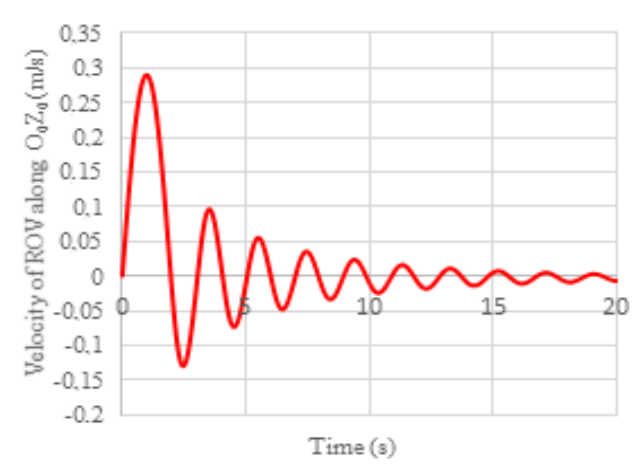

b)

Fig. 3 Velocity of ROV: a) component $V_{x}$ along $\left.O_{0} X_{0}, b\right)$ component $V_{z}$ along $O_{0} Z_{0}$

The evolution of the ROV displacement along the $O_{0} X_{0}$ axis is shown in Fig. 4. The maximal displacement is about $32 \mathrm{~m}$ after $20 \mathrm{~s}$. 


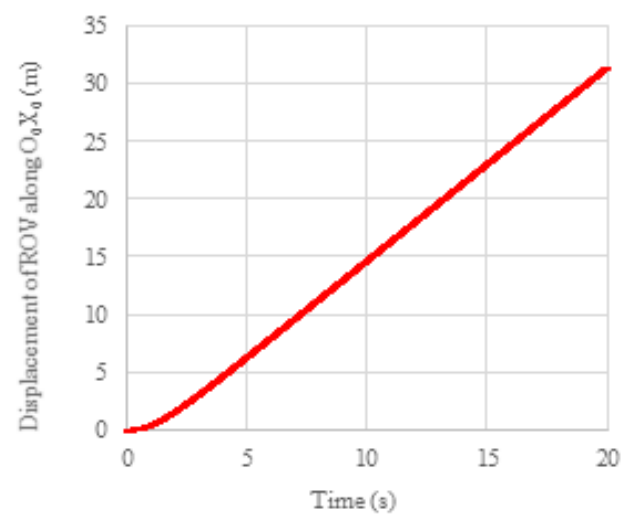

Fig. 4 Displacement of $R O V$ along $O_{0} X_{0}$ axis

The angle $\theta$ oscillations of the ROV around the $O_{0} Y_{0}$ axis are quite small, as it can be seen in Fig. 5 .

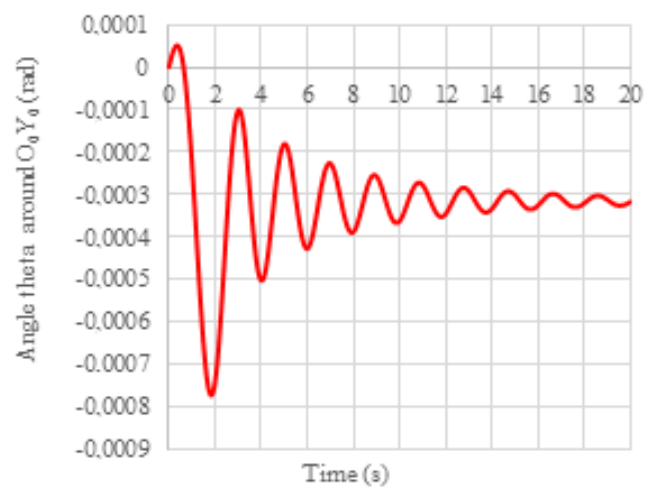

Fig. 5 Angle $\theta$ of $R O V$ around $O_{0} Y_{0}$ axis

Nevertheless, as illustrated in Fig. 6, the motion of the ROV around $O_{0} Z_{0}$ axis oscillates quite a lot during the first 10 seconds. After that, the motion of the ROV is less fluctuant and tends to being stable.

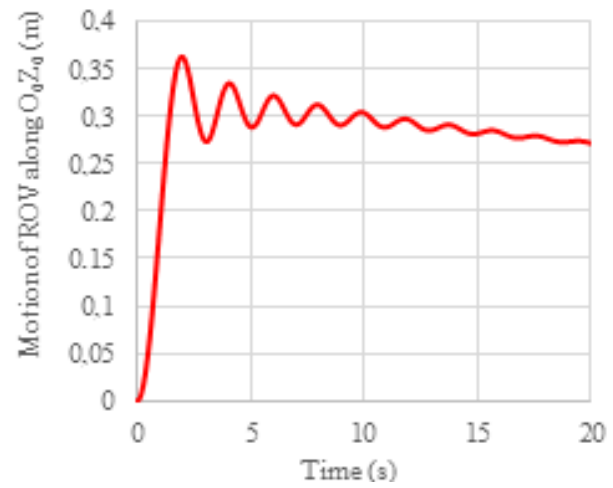

Fig. 6 Motion of $R O V$ around $O_{0} Z_{0}$ axis 


\section{Verification of the ROV Motion Model}

The computational results of the ROV motion are compared with the measured values. Experiments were held in an experimental water basin. A photo of the ROV prototype is shown in Fig. 7 and the schematic diagram of the experimental setup is shown in Fig. 8.

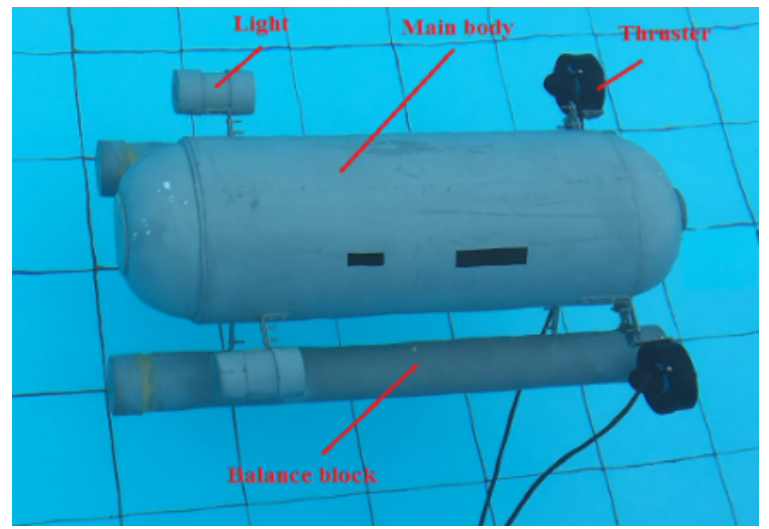

Fig. 7 ROV prototype

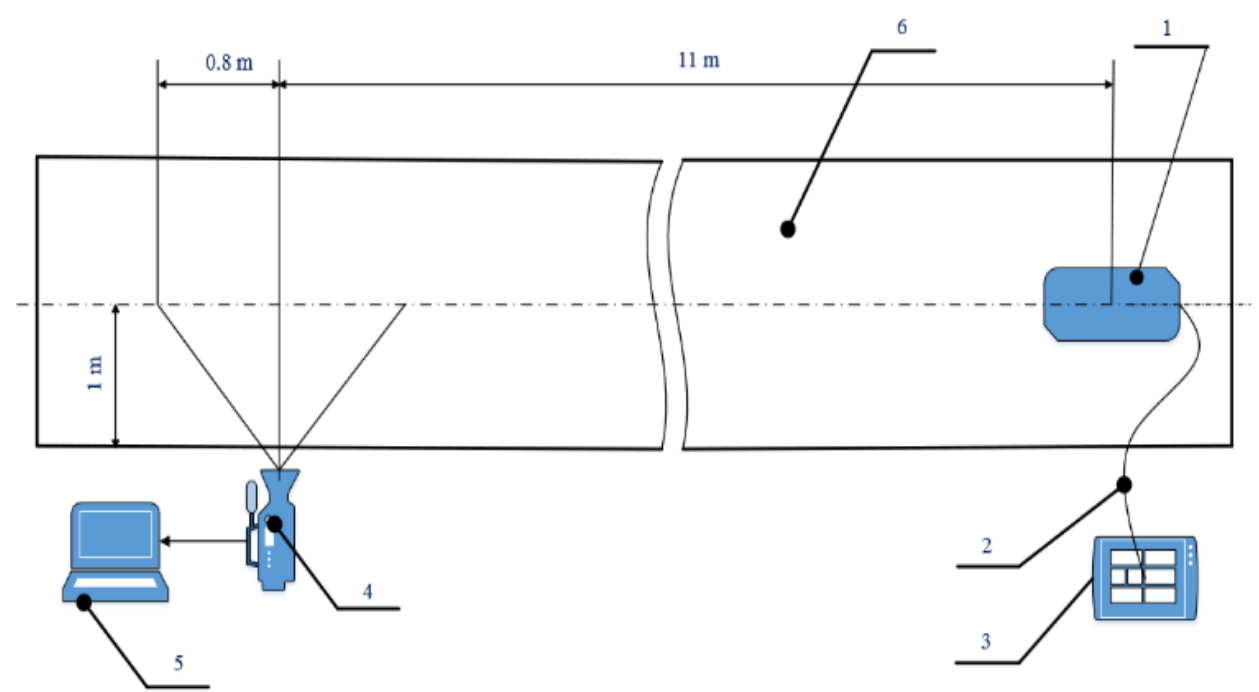

Fig. 8 Schematic diagram of the ROV experimental setup: $1-R O V$ prototype, $2-$ connecting cable, 3 - ROV control system, 4 - camera, 5 - computer, 6 - water basin

Velocities of the ROV prototype are measured by the high-speed camera FastCam SA 1.1 Model 675K-C1. The measuring laptop uses the data processing software TEMA.

This experiment enables to determine the ROV velocity $V_{x}$ along $O_{0} X_{0}$ axis in the period of its stable motion, i.e. the maximum velocity of the ROV. The range of measurements includes four cases of propulsive forces $(5,10,15,20 \mathrm{~N})$ for two distances 
between thrusters $(0.455 \mathrm{~m}$ and $0.505 \mathrm{~m})$. The propulsive forces are the same for both of the thrusters.

The comparison of some results of the developed ROV motion model and the measured values from the presented experiment for two distances between thrusters is given in Tab. 2.

Tab. 2 Comparison of ROV velocities for two distances between thrusters

\begin{tabular}{|c|c|c|c|c|}
\hline \multirow{2}{*}{$\begin{array}{c}\text { No. } \\
\text { I }\end{array}$} & \multirow{2}{*}{$\begin{array}{c}\text { Thruster force (each) } \\
\tau_{p}[\mathrm{~N}] \\
\text { Distance between } \\
\text { thrusters } L_{1}=0.455 \mathrm{~m}\end{array}$} & \multicolumn{2}{|c|}{$\begin{array}{c}\text { ROV velocity along } \boldsymbol{O}_{\mathbf{0}} \boldsymbol{X}_{\mathbf{0}} \\
V_{x}[\mathrm{~m} / \mathrm{s}] \\
\end{array}$} & \multirow{2}{*}{$\begin{array}{c}\text { Difference } \\
{[\%]}\end{array}$} \\
\hline & & Model & Experiment & \\
\hline 1 & 5 & 0.8300 & $0.7759 \pm 0.0103$ & 6.97 \\
\hline 2 & 10 & 1.1965 & $1.1261 \pm 0.0044$ & 6.25 \\
\hline 3 & 15 & 1.3987 & $1.2758 \pm 0.0114$ & 9.63 \\
\hline 4 & 20 & 1.6526 & $1.5109 \pm 0.0008$ & 9.38 \\
\hline II & $\begin{array}{c}\text { Distance between } \\
\text { thrusters } L_{1}=0.505 \mathrm{~m}\end{array}$ & Model & Experiment & \\
\hline 1 & 5 & 0.8300 & $0.7971 \pm 0.0042$ & 4.13 \\
\hline 2 & 10 & 1.1965 & $1.1636 \pm 0.0046$ & 2.83 \\
\hline 3 & 15 & 1.3987 & $1.3515 \pm 0.0032$ & 3.49 \\
\hline 4 & 20 & 1.6526 & $1.5616 \pm 0.0026$ & 5.83 \\
\hline
\end{tabular}

The comparison in the Tab. 2 shows that the ROV velocities $V_{x}$ increase with increasing distance between the thrusters. The ROV velocities computed by the ROV motion model are larger than those determined from the experiment. It is reasonable for the ROV operation under real conditions. The percentage of difference between the computational and experimental results is quite small (between $2.83 \%$ and $9.63 \%$ ). These results prove that the theoretical method of solution and the ROV motion model are appropriate.

\section{Conclusion}

A mathematical model has been developed to study the motion characteristics of the complex-shaped ROV with two planes of symmetry and one asymmetric plane. This model includes, besides the motion equations, hydrodynamic damping parameters and forces acting upon the ROV. The set of equations and matrices presented in this article was solved by the Runge-Kutta method using the Fortran programing language.

The mathematical model was validated for the given configuration of the examined ROV, consisting of the main body, two lights, two thrusters and two balance flotation blocks. Furthermore, the model was experimentally verified through measuring the ROV velocity by a high-speed camera. The presented model provides a satisfactory agreement with the measured ROV velocity.

\section{Acknowledgement}

The authors gratefully acknowledge the financial support from the NVCC03.03/20-20 funds of the VAST and the institutional funding of the University of Defence in Brno DZRO K 201. 


\section{References}

[1] GOMES, R.M.F., SOUSA, J.B. and PEREIRA, F.L. Modeling and Control of the IES Project ROV. In Proceeding of the European Control Conference. Cambridge: IEEE, 2003, p. 3424-3429. DOI 10.23919/ECC.2003.7086570.

[2] MARZBANRAD, A., SHARAFI, J., EGHTESAD, M. and KAMALI, R. Design, Construction and Control of a Remotely Operated Vehicle. In Proceeding of the ASME 2011 International Mechanical Engineering Congress and Exposition. Denver: ASME, 2011, p. 1295-1304. DOI 10.1115/IMECE2011-65645.

[3] EIDSVIK, O. and SCHJØLBERG, I. Determination of Hydrodynamic Parameters for Remotely Operated Vehicles. In Proceedings of the ASME $201635^{\text {th }}$ International Conference on Ocean, Offshore and Arctic Engineering. Busan: ASME, 2016, p. 1-10. DOI 10.1115/OMAE2016-54642.

[4] ISA, K., ARSHAD, M.R. and ISHAK, S. A Hybrid-Driven Underwater Glider Model, Hydrodynamic Estimation, and an Analysis of the Motion Control. Ocean Engineering, 2014, vol. 81, p. 111-129. DOI 10.1016/j.oceaneng.2014.02.002.

[5] LIANG, X., PANG, Y., WAN, L. and WANG, B. Dynamic Modelling and Motion Control for Underwater Vehicles with Fins. In Underwater Vehicles, Vienna: InTech, 2009, p. 539-556. ISBN 978-953-7619-49-7.

[6] FABER, T. Fluid Dynamics for Physicists. Cambridge: Cambridge University Press, 2010. 472 p. ISBN 978-0-521-42969-6.

[7] POLYANIN, A.D., KUTEPOV, A.M., VYAZMIN, A.V. and KAZENIN, D.A. Hydrodynamics, Mass and Heat Transfer in Chemical Engineering. Boca Raton: CRC Press, 2001. 408 p. ISBN 0-415-27237-8.

[8] FOSSEN, T.I. Handbook of Marine Craft Hydrodynamics and Motion Control. Chichester: Wiley, 2011, 596 p. ISBN 978-1-119-99149-6. 